\title{
Chirality tunneling and quantum dynamics for domain walls in mesoscopic ferromagnets
}

\author{
E. G. Galkina, ${ }^{1,2}$ B. A. Ivanov, ${ }^{1,3, *}$ Sergey Savel'ev, ${ }^{1,4}$ and Franco Nori ${ }^{1,5}$ \\ ${ }^{1}$ Frontier Research System, The Institute of Physical and Chemical Research (RIKEN), \\ Wako-shi, Saitama, 351-0198, Japan \\ ${ }^{2}$ Institute of Physics, 03028, Kiev, Ukraine \\ ${ }^{3}$ Institute of Magnetism, 03142 Kiev, Ukraine \\ ${ }^{4}$ Department of Physics, Loughborough University, \\ Leicestershire LE11 3TU, United Kingdom \\ ${ }^{5}$ Department of Physics, Center for Theoretical Physics, \\ Applied Physics Program, Center for the Study of Complex Systems, \\ University of Michigan, Ann Arbor, MI 48109-1040, USA
}

(Dated: October 30, 2018)

\begin{abstract}
We studied the quantum dynamics of ferromagnetic domain walls (topological kink-type solitons) in one dimensional ferromagnetic spin chains. We show that the tunneling probability does not depend on the number of spins in a domain wall; thus, this probability can be large even for a domain wall containing a large number of spins. We also predict that there is a strong interplay between the tunneling of a wall from one lattice site to another (tunneling of the kink coordinate) and the tunneling of the kink topological charge (so-called chirality). Both of these elementary processes are suppressed for kinks in one-dimensional ferromagnets with half-integer spin. The dispersion law (i.e., the domain wall energy versus momentum) is essentially different for chains with either integer or half-integer spins. The predicted quantum effects could be observed for mesoscopic magnetic structures, e.g., chains of magnetic clusters, large-spin molecules, or nanosize magnetic dots.
\end{abstract}

PACS numbers: 75.10.Jm, 03.75.Kk 


\section{INTRODUCTION}

Domain walls play an important role in the physics of magnets. For macroscopic bulk magnetic samples, domain walls, being extended classical objects, determine the demagnetizing processes, see, e.g., Refs. 1,2,3. For one-dimensional magnets, domain walls (kink-type solitons) play a different role: they are nonlinear excitations responsible for the destruction of long-range order. Thus, domain walls should be taken into account together with the linear excitations (magnons) $\stackrel{4}{\underline{4}} \underline{\underline{5}}$ Quantum properties are inherent to kinks in one-dimensional magnets (spin chains) with small spin values, like $S=1 / 2$ or $S=1$ and high anisotropy.

Classical solitons in one-dimensional Heisenberg ferromagnets have been investigated in detail. For continuum media, their dynamical properties are determined by the LandauLifshitz equation for the magnetization vector $\mathbf{m}(x, t)$, where $\mathbf{m}^{2}=1$, see e.g., Refs. $\underline{3}$, 4,6. For such systems, kink-type solitons can be treated as classical particle-like objects. However, kinks are extended objects, and for spin chains with low anisotropy $K \ll J(J$ is the exchange integral and $K$ the anisotropy constant), a kink involves a large number

of spins $N_{\text {kink }} \sim S \sqrt{J / K} \gg 1$. For this reason, domain walls for low-anisotropy magnets should be formally considered as a mesoscopic, rather than a microscopic, object. Thus, it is not obvious whether or not the effects of quantum coherence are essential for domain wall dynamics in mesoscopic ferromagnets.

Artificial quasi-1D mesoscopic materials (including chains of small magnetic elements, such as small magnetic particles of nanometer size (magnetic dots), patterned magnetic films, magnetic clusters and high-spin molecules) are of great importance, $, \frac{7}{8}, 9,9,10,11,12,13,14$ and are promising elements for computers. $\stackrel{9}{ }$ These materials often manifest unique physical properties that are absent in bulk samples, for instance, macroscopic quantum coherence and quantum tunneling, see e.g., Refs. 7,8. These quantum properties of small magnetic systems could be potentially useful for quantum computing. $\frac{15}{}$

Quantum coherence can occur when states with the same energy are separated by a small potential energy barrier. Kink-type solitons can demonstrate a rich variety of different quantum effects. Indeed solitons are particle-like objects and their quantum dynamics include the tunneling of the kink coordinates through a potential barrier, separating equivalent positions in 1D chains. These tunneling effects are coherent, and, as for electrons in crystals, they lead to the formation of band spectra. 
Another type of tunneling involves the domain wall chirality. Namely, a domain wall is characterized by the deviation of the magnetization from the easy axis. The corresponding value of the total spin $\mathbf{S}_{\perp}$ is quite large, i.e., $\left|\mathbf{S}_{\perp}\right|$ is of the order of $S N_{\text {kink }} \gg 1$. For biaxial magnets, the domain wall state has two-fold degeneracy along the $\mathbf{S}_{\perp}$ direction. From a mathematical point of view, the sign of the quantity $\mathbf{S}_{\perp}$ corresponds to the value of topological charge or chirality $\chi= \pm 1$. Thus, the kink structure is doubly-degenerate over the sign of this topological charge, implying the possibility of a quantum coherent superposition of two states with different chirality.

The tunneling of topological charges has previously been discussed for different topological solitons (kinks, vortices, and disclinations) in antiferromagnets; for a review see $\underline{\underline{16}}$ It is worth noting here that the static distribution of the corresponding order parameters, the normalized magnetization vector $\mathbf{m}$ for ferromagnets $\left(\mathbf{m}^{2}=1\right)$ and sublattice magnetization vector $\mathbf{l}\left(\mathbf{l}^{2}=1\right)$ for antiferromagnets, are all identical. For antiferromagnetic spin chains, the rate of the chirality tunneling process appears to be unexpectedly high, because the tunneling exponent is of the order of the atomic spin $S$, being independent on the number of spins $N_{\text {kink }} \gg 1$ within the kink. ${ }^{17}$ Thus, quantum effects could be essential not only for literally 1D objects like spin chains, but also for mesoscopic antiferromagnetic samples, like thin antiferromagnetic wires. 18

The dynamic properties of ferromagnets are described by the Landau-Lifshitz equation for the vector $\mathbf{m}$ with no inertial term. This is in strong contrast with the inertial dynamics of antiferromagnets, described by the so-called sigma-model equation for the sublattice magnetization vector l, e.g., Refs. 4, 3. It might look a bit paradoxical that the quantum properties of ferromagnets are much more complicated than for both quantum antiferromagnets and quantum Josephson junctions $\frac{19,20,21}{}$. The reason is because within the sigma-model approach the dynamics of the vector $\mathbf{l}$ is similar to that of the usual inertial dynamics of a particle (strictly speaking, the dynamics of a particle along the surface of the sphere $\mathbf{l}^{2}=1$ ); whereas the Landau-Lifshitz Lagrangian contains a complicated Dirac-monopole term with non-trivial topological properties (Berry phase), see, e.g., Ref. 23. This circumstance leads to a number of subtle and intriguing effects, e.g., the suppression of tunneling transitions due to the interference of the instanton trajectories. $\underline{25}, \underline{26}$

An example of strikingly different quantum dynamics of ferromagnets and antiferromagnets is the tunneling chirality. For kinks in antiferromagnets, chirality tunneling is not 
correlated with the translational motion of kinks. $\underline{17} \underline{18}$ In contrast, some results known in the literature imply that for ferromagnets the situation can be different. Ref. 27 noted that unmovable kinks with different values of the chirality must have different values of the momentum. Ref. 28 pointed out that the chirality tunneling rate grows with the intensity of the kink spatial pinning. Ref. 29 showed that for a free (i.e., with no pinning) domain wall in a ferromagnet, the momentum of which is conserved, the tunneling of the chirality is prohibited.

In this article we develop a consistent quantum theory of domain walls in one-dimensional ferromagnets, with a complete description of all possible coherent quantum effects. We show that the tunneling of the chirality can be described as a tunneling in momentum space. This is closely connected with the tunneling of the kink coordinates; indeed, all these tunneling processes are naturally described within phase plane $(X, P)$.

The article is organized as follows. In the next section II, we introduce the Hamilton variables: kink coordinate $X$ and kink momentum $P$. In this section II we show how to consistently define the chirality of a kink via the value of kink momentum. A Hamiltonian approach, valid for describing both quantum effects, tunneling of kink coordinates and kink chirality, will be developed in the same Section III. Then, in Section III, specific tunneling effects will be analyzed based on this approach. Kink dispersion relations will also be derived there.

\section{HAMILTON DESCRIPTION OF KINK DYNAMICS}

In order to describe a one-dimensional system of mesoscopic magnetic particles allowing kink dynamics, we assume that each particle has an internal magnetic anisotropy, with the chosen axes to be parallel for all particles in a system. The geometry of the problem is shown in Fig. 1, We will also consider isotropic nearest neighbor interactions. Moreover we assume that any internal degrees of freedom of the particles can be neglected, and each particle can be treated as a single magnetic moment (spin).

Note that kinks in a ferromagnet with pure axial symmetry $\mathrm{C}_{\infty}$ cannot move because the

projection of the total spin on the easy axis $S_{3}^{(\mathrm{tot})}$ should change while the kink is moving. However, the Hamiltonian is invariant under rotations around the easy-axis and it commutes with $S_{3}^{\text {(tot) }}$, prohibiting such dynamics. In order to allow kink dynamics, we can consider the 


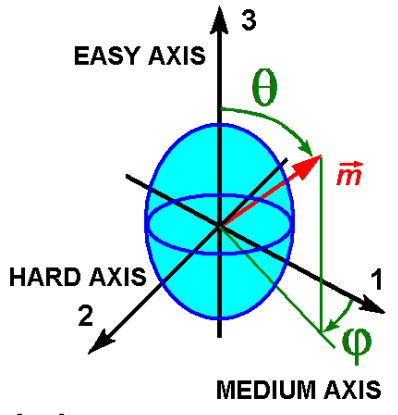

(a) (b)

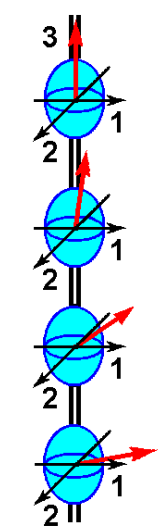

FIG. 1: The geometry of the problem; $a$ ) anisotropy axis for a single magnetic particle; $b$ ) a schematic representation of a chain of particles. Each particle is represented by its spin.

two-fold magnetic anisotropy. This is in contrast with antiferromagnets, where kinks can move even for purely uniaxial anisotropy, with an easy axis of $\mathrm{C}_{\infty}$ symmetry.

\section{A. Model}

The Hamiltonian of a chain-like system of magnetic particles can be written in the same form as for a discrete ferromagnetic chain (one-dimensional lattice with atomic spacing $a$ ) with a spin operator $\mathbf{S}_{n}$ located in each lattice site $n$,

$$
\mathcal{H}=-J \sum_{n} \mathbf{S}_{n} \cdot \mathbf{S}_{n+1}+\sum_{n}\left[K_{1} S_{1, n}^{2}+K_{2} S_{2, n}^{2}\right]
$$

Here the first term describes the isotropic exchange interaction of spins, and the second sum corresponds to the two-fold magnetic anisotropy. We have chosen $K_{2}>K_{1}>0$, so that the orthogonal axes 3,1 and 2 are the easy axis, the medium axis and the hard axis, respectively. We consider each spin operator to be a classical vector with constant modulus $\mathbf{S}=S \mathbf{m}$ and unit vector $\mathbf{m}$ (i.e., this vector points in the unit sphere $\mathbf{m}^{2}=1$ ). The dynamics of the variables $\mathbf{m}_{n}$ is governed by the discrete version of the Landau-Lifshitz equation,

$$
\hbar S \frac{\partial \mathbf{m}_{n}}{\partial t}=\left(\mathbf{m}_{n} \times \frac{\partial W}{\partial \mathbf{m}_{n}}\right)
$$

where $W \equiv W\left(\mathbf{m}_{n}\right)$ is the energy of the considered ferromagnetic chain. The dynamics of the variable $\mathbf{m}_{n}$, for a given point $n$ in a lattice, is determined by the Lagrangian,

$$
\mathcal{L}=-\hbar S \sum_{n} \frac{\mathbf{n}}{1+\left(\mathbf{n} \cdot \mathbf{m}_{n}\right)} \cdot\left(\mathbf{m}_{n} \times \frac{\partial \mathbf{m}_{n}}{\partial t}\right)-W
$$


where $\mathbf{n}$ is an arbitrary unit vector. For the continuum approximation, the set of variables $\mathbf{m}_{n}$ determined on the lattice sites $n$ should be replaced by a smooth function of the continuous coordinate $x: \mathbf{m}_{n} \rightarrow \mathbf{m}(x)$. In this continuum approximation, the Lagrangian takes the form

$$
\mathcal{L}=-\int d x\left(\mathbf{A} \cdot \frac{\partial \mathbf{m}}{\partial t}\right)-W, \mathbf{A}=\frac{\hbar S}{a} \cdot \frac{(\mathbf{n} \times \mathbf{m})}{1+(\mathbf{n} \cdot \mathbf{m})},
$$

where $W$ is an energy functional written by expanding over gradients of $\mathbf{m}$; see, for example, Ref. 24. The vector $\mathbf{A}$ has the form of a vector-potential of a Dirac monopole field, i.e., the curl of $\mathbf{A}$ over the variable $\mathbf{m}$ is proportional to $\mathbf{m}$ :

$$
\operatorname{curl}_{\mathbf{m}} \mathbf{A}=\hbar S \mathbf{m} / a
$$

see, for example, Ref. 23. The vector potential has a singularity (Dirac string) for $(\mathbf{m} \cdot \mathbf{n})=$ -1 , i.e., on a half-line in $\mathbf{m}$-space. It is important to note that the vector potential $\mathbf{A}$ is accurate within some gauge transformations, which includes changing the direction of the Dirac string, but the Landau-Lifshitz equations containing $\operatorname{rot}_{\mathbf{m}} \mathbf{A}$ are invariant with respect to gauge transformations. ${ }^{23}$ It is worth mentioning here that for antiferromagnets within the sigma-model approach, the dynamical part of the Lagrangian has a standard inertial term

$$
L_{\mathrm{dyn}, \mathrm{AFM}} \propto\left(\frac{\partial \mathbf{l}}{\partial t}\right)^{2},
$$

in contrast to $\mathbf{A} \cdot(\partial \mathbf{m} / \partial t)$ for ferromagnets.

It is convenient to represent the unit vector field $\mathbf{m}$ by two independent angular variables $\theta$ and $\varphi$,

$$
m_{1}=\sin \theta \cos \varphi, m_{2}=\sin \theta \sin \varphi, m_{3}=\cos \theta .
$$

In terms of these variables, the Landau-Lifshitz equation (2) takes the form

$$
\begin{aligned}
\frac{S \hbar}{a} \sin \theta \frac{\partial \theta}{\partial t} & =\frac{\delta W}{\delta \varphi}, \\
\frac{S \hbar}{a} \sin \theta \frac{\partial \varphi}{\partial t} & =-\frac{\delta W}{\delta \theta},
\end{aligned}
$$

where $W \equiv W\{\theta, \varphi\}$ is the energy functional written it terms of the field variables $\theta$ and $\varphi$. The Hamiltonian (11) can be rewritten as

$$
W\{\theta, \varphi\}=\int \frac{d x}{a}\left\{\frac{J a^{2}}{2}\left[\left(\frac{\partial \theta}{\partial x}\right)+\sin ^{2} \theta\left(\frac{\partial \varphi}{\partial x}\right)^{2}\right]+\sin ^{2} \theta\left[K_{1}+\left(K_{2}-K_{1}\right) \sin ^{2} \varphi\right]\right\}
$$


where the non-zero difference $\left(K_{2}-K_{1}\right)$ determines the anisotropy in the basal plane.

The Landau-Lifshitz equations have an obvious integral of motion, the energy $W$. In the continuum approximation, translational invariance leads to the conservation of the linear momentum of the magnetization field $P$ (momentum, for short). The expression for the momentum is determined ${ }^{3,6}$ by the dynamical part of the Lagrangian

$$
P=\int d x\left(\mathbf{A} \cdot \frac{\partial \mathbf{m}}{\partial x}\right) .
$$

As noted above, the vector-potential $\mathbf{A}$ is known up to a gauge transformation, and the momentum $P$ depends on the gauge used for $\mathbf{A}$. This is one of the problems for describing the dynamics of solitons in ferromagnets. This problem does not exist for antiferromagnets, where the momentum is proportional to the integral $\int d x(\partial \mathbf{l} / \partial x)(\partial \mathbf{l} / \partial t)$. However, it turns out that the relative momentum for any pair of kinks in a ferromagnet can be uniquely determined. Thus, the kink momentum is accurate within the position of the origin in $P$-space; that is, the choice of a kink assigned with the value of $P=0$.

\section{B. Topological analysis of domain wall structure}

For a kink in a ferromagnetic chain, the values of the on-site variables $S_{3, n}$ have opposite values in front and behind of the kink: $S_{3, n} \rightarrow \pm S$ at $n \rightarrow \pm \infty$. In other words, a kink can be seen as a path connecting the poles of the sphere $\mathbf{m}^{2}=1$, corresponding to the two easy directions of magnetization space (see Fig. 2). For definiteness, we assume that $m_{3}=1$ at $x \rightarrow-\infty$ and $m_{3}=-1$ at $x \rightarrow+\infty$ (see Fig. 2). For ferromagnets with non-zero anisotropy in the basal plane, $\partial W / \partial \varphi \neq 0$, a domain wall can move with some velocity $v$ smaller than the limit value $v_{c}$. Within the continuum approximation, such moving domain walls are described by a simple traveling-wave one-soliton solution of the Landau-Lifshitz equation (6) of the form $\theta=\theta(\xi), \varphi=\varphi(\xi)$, with $\xi=x-v t$. However, it is hard to find an analytical solution of a set of two second-order equations of this type, and we will start with a qualitative analysis.

Due to symmetry, there are two types of stationary domain walls, with $\theta=\theta(x)$ and $\varphi=$ const. For these domain walls, the vector $\mathbf{m}$ turns either within the easy plane $(3,1)$, for the first type of walls, or within the hard plane $(3,2)$, for the second one. The trajectories describing these domain walls are denoted by symbols $B+, B-$ and $N_{1}, N_{2}$, respectively, on 

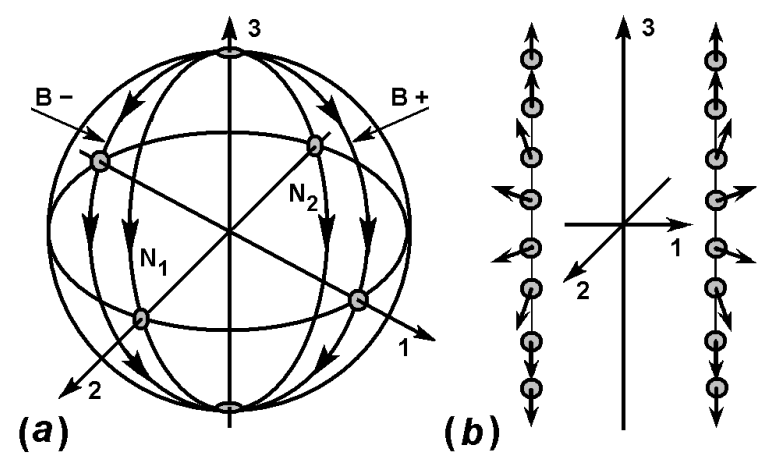

FIG. 2: (a) Trajectories on the sphere $\mathbf{m}^{2}=1$ (the direction of motion is indicated by the arrows) corresponding to different kinks; the labels 1, 2, and 3 indicate the anisotropy axes of the magnet. Two of the most favorable kinks with chirality $\chi= \pm 1$ are shown by the symbols $B+$ and $B-$; unfavorable kinks with indefinite chirality are labeled by $N_{1}$ and $N_{2}$. The crossings between the trajectories and the axes 1 or 2 are shown by four gray ovals in (a). The trajectories $N_{1}$ and $N_{2}$ divide the sphere in two domains, associated with kinks of different chirality, as discussed in the text. (b) Spin distribution for favorable kinks with chirality $\chi= \pm 1$.

the Fig. 2, Their energies are $E_{1}$ for $B \pm$ and $E_{2}>E_{1}$ for $N_{1,2}$. Other domain walls having $v \neq 0$ and energies $E_{1}<E(v)<E_{2}$, are described by paths, located in between these chosen trajectories on the sphere.

The kink momentum is the total momentum of the magnetization field, calculated along the corresponding solution of the Landau-Lifshitz equation. $\underline{3}^{\underline{6}}$ For domain walls, it can be written in the form of an integral around the contour of the sphere Fig. 2, depicting a kink, $P=\int \mathbf{A}(\mathbf{m}) d \mathbf{m}$, there $\mathbf{A}$ is the vector-potential of the Dirac monopole field (4). A difference of the momentum values for two different kinks can be described as an integral along a closed contour. It can be written through a surface integral of the type of $\int d \mathbf{S} \operatorname{rot}_{\mathbf{m}} \mathbf{A}$ and it is equal to $\hbar S / a$, multiplied by the area, on the sphere $\mathbf{m}^{2}=1$, inside two trajectories, corresponding to these pairs of kinks. ${ }^{33}$ It is clear that for a biaxial ferromagnet there are pairs of diametrically opposite trajectories (e.g., the trajectories $B+$ and $B-$ in Fig. 2) corresponding to energetically equivalent but physically different kinks. For these pairs of kinks, the closed path borders half of a sphere, with an area of $2 \pi$, and the momentum difference equals $2 \pi \hbar S / a$. Hence we can readily obtain the periodic dependence of the kink 
energy on its momentum with the period $P_{0}$,

$$
P_{0}=\frac{2 \pi \hbar S}{a}
$$

For those kinks which are close to the most favorable kink $B+(B-)$, the value of the momentum $P\left(\Delta P=P-P_{0}\right)$ is small; thus, the parabolic approximation can be used and the energy can be written as $E=P^{2} / 2 M$ (or $\left.E=(\Delta P)^{2} / 2 M\right)$, where $M$ is the effective mass of the kink. For the model (8) , the effective mass $M$ takes the value of the well known Döring effective mass, obtained as early as the 1930's; see, e.g., Refs. 2,6. This effective mass turns to infinity when $\left(K_{2}-K_{1}\right) \rightarrow 0$. This is another indication that in a pure uniaxial model of a ferromagnet $\left(K_{2}=K_{1}\right)$, domain wall motion is impossible. However there is no contradiction between the finite value of $P \propto \varphi$ and the condition $v=0$ : if $P=M v$, then the momentum can be finite when $M \rightarrow \infty$ and $v \rightarrow 0$.

Coherent tunneling assumes the presence of at least two different states having the same energy; for instance, the two states of kinks in a biaxial ferromagnet with different values of the topological charge. A topological classification ${ }^{34}, 35$ of kinks can be done in the same way for both ferromagnets and antiferromagnets. First, the difference of values of the magnetization vector $\mathbf{m}_{x}$ (or $\mathbf{l}_{x}$, for antiferromagnets) on the right and on the left sides of the kink determine the topological charge $\pi_{0}$ of the kink. Changing this topological charge requires overcoming the potential barrier, proportional to the system size (formally, infinite barrier) that cannot be realized by tunneling. Second, a topological charge of the type $\pi_{1}$ is determined by mapping the coordinate space of the spin chain (the line $-\infty<x<\infty$ ) onto the circle $\left\{m_{1}^{2}+m_{3}^{2}=1, m_{2}=0\right\}$, situated in the easy-plane of the ferromagnet. The appearance of two topological charges of different levels can be formally described using the relative homotopy group, as discussed in Ref. 35 .

The $\pi_{1}$ charge is described by the integral

$$
\chi=\frac{1}{\pi} \int_{-\infty}^{\infty}\left(\mathbf{e}_{2}\left(\mathbf{m} \times \frac{\partial \mathbf{m}}{\partial x}\right)\right) d x .
$$

In other words, the chirality $\chi= \pm 1$ determines the sense of rotation (clockwise or counterclockwise) of $\mathbf{m}$ along the chain. This standard definition of the chirality used in Refs. 27, 28,29 is only valid for kinks located on the unit sphere near the most energetically favorable configurations $B+$ and $B-$, as shown in Fig. 2; that is, for kinks having small velocities $v$. For this case, the effective mass approximation is valid, and these two 
kinks are well separated. According to the topological analysis, these kinks have different values of the chirality $\chi= \pm 1$; and within a self-consistent Hamiltonian approach, they correspond to different values of the momentum: $P=0$ and $P=P_{0}$. However, for kinks moving with a non-small velocity and with arbitrary values of the momentum, the above definition of the chirality should be modified.

For treating the whole order parameter space (i.e., the sphere $\mathbf{m}^{2}=1$ ), kinks with $\chi= \pm 1$ can be transferred to each other through energetically unfavorable kinks of type $N_{1}$ or $N_{2}$, schematically shown in Fig. 2. Here the barrier is finite (and equal to $E_{2}-E_{1}$; which is large when $K_{2} \gg K_{1}$ ) and the process of kink chirality $\chi$ tunneling is possible. The concept of chirality, as a discrete number $\chi= \pm 1$, is naturally connected with the presence of a discrete degeneracy in the dependence of the kink energy on its momentum. The discrete parameter $\chi= \pm 1$ determines one of two different, but energetically equivalent, kink states existing in a biaxial ferromagnet. The values $\chi=1$ and $\chi=-1$ can be naturally attributed to kink states with trajectories in two equivalent semi-spheres, $m_{1}>0$ and $m_{1}<0$, respectively. The chirality value is not determined for the unfavorable static kinks only (Neel walls) for which the trajectories $N_{1}$ and $N_{2}$ pass through the hard axis. In this sense, chirality tunneling can be seen as a tunneling effect in momentum space with a non-small (of the order $\left.P_{0}=2 \pi \hbar S / a\right)$ change of the kink momentum.

\section{Moving domain wall structure}

To confirm the general features for moving domain walls discussed above, we will discuss an exact solution of (6) known for the model of biaxial ferromagnets with the energy (8). It is easy to find the structure of domain walls with zero velocity. There are two types

of domain walls having thickness $x_{1,2}=a \sqrt{J / 2 K_{1,2}}$ and energies $E_{1,2}=2 S \sqrt{2 J K_{1,2}}$ with $E_{1}<E_{2}$. These are the one-dimensional analogs of the usual Bloch and Neel domain walls; see Refs. 2, 6 .

The structure of a domain wall moving with a non-small velocity within the model (8) was obtained by Walker at the end of the 1950s, see Refs. 2, 3, 6. For this solution, the function $\theta=\theta(\xi), \xi=x-v t$, and the value of $\varphi=\varphi_{0}=$ const is independent on $\xi$. The 
value of $\varphi_{0}$ is determined by the domain wall velocity $v$ as follows

$$
\frac{v \hbar}{a \sqrt{J K}}=q \frac{\varepsilon \sin \varphi \cos \varphi}{\sqrt{1+\varepsilon \sin ^{2} \varphi}}
$$

here and below we use the notation $\varepsilon=\left(K_{2}-K_{1}\right) / K_{1}$ to shorted the expressions. The relation (11) governs, in particular, the maximal possible value of a domain wall velocity, the so-called Walker velocity $v_{W}$

$$
v_{W}=(a / \hbar) \sqrt{J K}(\sqrt{1+\varepsilon}-1) .
$$

It is interesting to note that $v_{W}$ is smaller than the minimal phase velocity of spin waves,

$$
v_{\mathrm{ph}}=(a / \hbar) \sqrt{J K}(\sqrt{1+\varepsilon}+1) .
$$

The values of $v_{W}$ and $v_{\mathrm{ph}}$ coincide only in the limit $\varepsilon \rightarrow \infty$, when the Landau-Lifshitz equation (6) can be mapped onto the sine-Gordon equation. However, if $\varepsilon$ is small, then

$$
v_{W} \simeq \varepsilon v_{\mathrm{ph}} / 4 \ll v_{\mathrm{ph}}
$$

The value of $v_{W}$ vanishes when $\varepsilon \rightarrow 0$; that is, as was noted above, the domain wall cannot move at all for pure uniaxial ferromagnets.

The Walker's solution can be presented in the explicit analytical form

$$
\cos \theta=q \cdot \tanh \left[\frac{\xi}{x_{0}(v)}\right], \sin \theta=\sigma\left\{\cosh \left[\frac{\xi}{x_{0}(v)}\right]\right\}^{-1},
$$

where $q= \pm 1, \sigma= \pm 1$

$$
x_{0}(v)=a \sqrt{\frac{J}{2 K_{1}\left(1+\varepsilon \sin ^{2} \varphi\right)}}
$$

and $\varphi=\varphi(v)$ is determined by Eq. (11). The two topological charges introduced above naturally appear here as the quantities $q$ and $\sigma ; q= \pm 1$ determines the $\pi_{0}$-topological charge of the kink, and $\sigma= \pm 1$ governs the spin direction in the kink center and it is naturally connected with the $\pi_{1}$-topological charge, the chirality $\chi$.

A straightforward calculation of the kink energy leads to the formula

$$
E=E_{1} \sqrt{1+\varepsilon \sin ^{2} \varphi}, E_{1}=2 S^{2} \sqrt{2 J K_{1}}
$$

where $\varphi=\varphi(v)$ is determined by Eq. (11). The $E(v)$ dependence consists of two branches. In three-dimensional ferromagnets, where two-dimensional plane domain walls are present, 
the upper branch of the domain walls is unstable. However this instability is developed via non-uniform perturbations in the domain wall plane, and such fluctuations do not exist for domain walls in one-dimensional magnets. Below we will show that for the more natural domain wall energy representation (namely, as a function of its momentum) the $E(P)$ function is single-valued.

For the Walker solution, the value of $\varphi$ is $\xi$-independent, $, 2,3$ and the paths on the sphere present at Fig. 2 are the halves of the big circles passing through the poles of the sphere. Therefore, the domain wall momentum can be written as

$$
P=P_{0} \frac{\varphi}{\pi}
$$

and the explicit form of the dependence of the domain wall energy on its momentum can be rewritten as

$$
E=E_{1} \sqrt{1+\frac{2 T_{0}}{E_{0}} \sin ^{2} \frac{\pi P}{P_{0}}}, \quad \frac{2 T_{0}}{E_{1}}=\frac{K_{2}-K_{1}}{K_{1}}
$$

where

$$
E_{1}=2 S^{2} \sqrt{2 J K_{1}}
$$

is the minimum energy of the domain wall introduced above. It is worth noting that the equation (17) reproduces the dependence $v(\varphi)$ (11) within the Hamilton dynamics,

$$
\frac{d X}{d t} \equiv v=\frac{\partial H(X, P)}{\partial P} .
$$

This leads to unusual dynamical features, like the oscillatory motion of a domain wall as a response to a dc driving force (e.g., dc magnetic field parallel to the easy axis). These peculiarities are well-known for the exact Walker solution and have been experimentally

established for moving domain walls in magnetic bubble materials; see, e.g. Ref. 2. Here we were able to write down the explicit form of the function $E(P)$, but the periodic dependence $E(P)$ with the same value of $P_{0}$ is present for any continuum model of a ferromagnet with biaxial anisotropy. Such periodic dependence $E(P)$ is also valid for biaxial discrete models; for details, see Ref. 32 .

\section{Kink coordinate and lattice pinning}

As we will show, the quantum properties of kinks can be described within a semiclassical analysis of the Hamilton dynamics of collective variables: the kink coordinate $X$ and 
conjugated momentum $P$. This dynamics is determined by the characteristic Hamilton function $H(P, X)$. In a continuous approximation, the definition of kink coordinate is obvious. However, the Hamilton relation

$$
\frac{d P}{d t}=-\frac{\partial H(P, X)}{\partial X}
$$

shows that the kink momentum is conserved for any model with the Hamiltonian independent on $X$. Therefore any processes of tunneling in momentum space (in particular, the tunneling of the domain wall chirality) can only occur if the Hamilton function $H$ depends on the domain wall position $X$. For our model, such dependence can only be caused by a lattice pinning of the kink. Thus, for a consistent description of quantum tunneling, lattice pinning must be considered.

A first step in this direction is to define the domain wall coordinate $X$ treated as a collective variable and conjugated to the kink momentum $P$. The kink coordinate $X$ in the discrete model can be naturally determined through the spin operators,

$$
X=\frac{a}{2 S} \sum_{n}\left[S_{3, n}-S_{3, n}^{(0)}\right]
$$

, where $S_{3, n}^{(0)}$ corresponds to a certain "reference" kink, $\frac{22}{2}$ which coordinate is chosen as $X=0$. The total spin projection onto the axis 3 is conserved, $S_{3}^{\text {tot }}=\sum_{n} S_{3}=$ const; thus, for uniaxial ferromagnets with $K_{2}=K_{1}, d X / d t=0$ and a kink dynamics is impossible. Another consequence appears when taking into account the Hamilton relation $d X / d t=$ $\partial H(P, X) / \partial P$. For the purely uniaxial case, $H(P, X)$ does not depend on $P$. All of these general considerations here are characteristic of the exact Walker solution.

A spin configuration corresponding to a kink with a specified value of the coordinate $X$ can be obtained by minimizing the Hamiltonian with respect to the variables $\theta_{n}$ and $\varphi_{n}$, for a fixed value of the total spin $S_{3}^{(\text {tot })}$. To do this, we use a procedure proposed and numerically realized for the analysis of different dynamical solitons, $\underline{\underline{30}}$ which are described by a conditional minimum of a discrete spin Hamiltonian. Using this method one can easily determine the structure of the kink and obtain the dependence of the kink energy on its coordinate $X$; this for finite spin chains described by any classical spin Hamiltonian.

Now, we consider model (1) with a purely single-ion anisotropy, for a finite chain of size $N_{c}$, with boundary conditions $\cos \theta_{n}=1$ and $\cos \theta_{n}=-1$ at different ends of the chain. The size of the chain $N_{c}$ is chosen to be much larger than the width of the kink. In particular, 


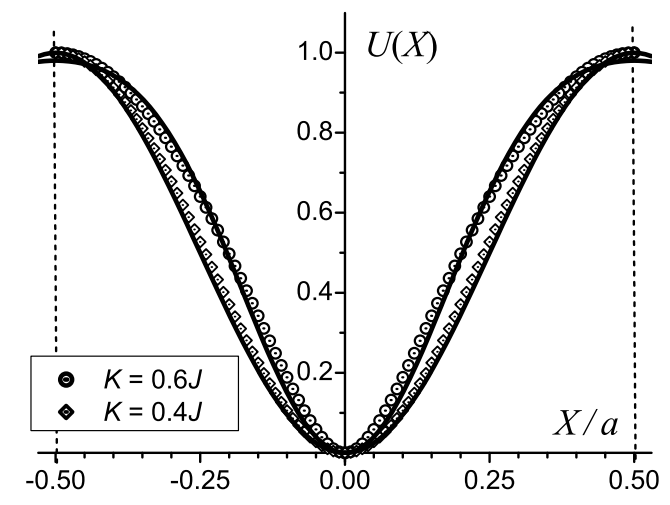

FIG. 3: Shape of the domain wall pinning potential $U(X)$, normalized by its maximal value $U(a / 2)$, for two values of the anisotropy constant. Symbols denote the numerical data. The fit for the model dependence (19) is shown by the full line.

for a reasonable anisotropy $K>0.2 J$, the kinks occur as well-localized excitations. As a result, the kink energy is independent of $N_{c}$ for $N_{c} \geq 30$. For extremely high values of the anisotropy, $K>K_{c}$, with $K_{c}=0.667 J$, the domain wall becomes purely collinear, $\underline{31}$ with all spins up or down, $S_{3}= \pm S$. For such collinear states, the continuum description of the domain wall dynamics and its topological analysis are obviously incorrect, and we should restrict our consideration to moderate values of the anisotropy: $0.2 J<K \leq 0.65 J<K_{c}$.

Considerable influence of the lattice pinning appear when $K \geq 0.25 \mathrm{~J}$. A more favorable position of the kink is between neighboring spins. Thus, the values $S_{3, n}$ at two neighboring spins are equal in magnitude and opposite in sign: $S_{3}= \pm S(0)<1$. Choosing the value of $X=0$ for one of such states, we can determine the pinning potential having equivalent minima at the points $X=a n$, where $n$ is an integer. The states with kink on a lattice site with $X=a(2 n+1) / 2$ correspond to maxima of the pinning potential, as shown in Fig. 3. In general, it can be concluded that, for moderate values of the anisotropy $K \leq K_{c}$, the pinning potential is not large compared to the "static" energy of the kink. The dependence $U(X)$ is fairly well described by the simple harmonic relation

$$
U(X)=U_{0} \cdot \sin ^{2}\left(\frac{\pi X}{a}\right)
$$

On the other hand, higher Fourier components are also present in the dependence $U(X)$, 


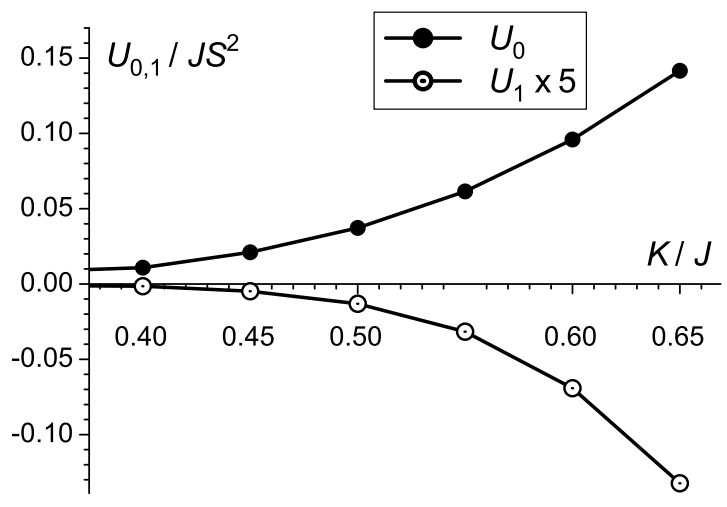

FIG. 4: Coefficients $U_{0}$ and $U_{1}$ (in units of $J S^{2}$ ) in Eq. (19) for some values of anisotropy constant $K_{1}$.

especially for higher anisotropy. For example, for a more general form

$$
U(x)=U_{0} \sin ^{2}\left(\frac{\pi X}{a}\right)+U_{1} \sin ^{2}\left(\frac{2 \pi X}{a}\right)
$$

the contribution of $U_{1}$ can be noticeable. The values of $U_{0}$ and $U_{1}$ as functions of the reduced anisotropy $K / J$ are presented in Fig. 4. In general, for higher values of the anisotropy $K$ one can see a broadening of the curve $U(X)$ near its maximum and, correspondingly, a narrowing of the curve near the minima. Note that when $K>K_{c}$, the function $U(X)$ has a cusp at $X=0$.

To conclude this section, we derive the periodic dependence of the domain wall Hamiltonian on both collective variables $X$ and $P$. Namely, to describe the quantum dynamics, we can use the Hamiltonian $H(P, X)=T(P)+U(X)$, where both functions $T(P)$ and $U(X)$ are periodic: $U(X)=U(X+a)$ and $T(P)=T\left(P+P_{0}\right)$.

\section{QUANTUM TUNNELING EFFECTS IN KINK DYNAMICS}

To describe the quantum dynamics of domain walls, we can proceed with the Hamiltonian $H(P, X)=T(P)+U(X)$, where the "kinetic energy" $T(P)$ is described by Eq. (17) and the "potential energy" is caused by a periodic lattice pinning potential $U(X)=U(X+a)$; see Eqs. (18) or (19). The most crucial point is the presence of the double periodicity of $H(P, X)$, with respect to both $X$ and $P$. For simplicity, below we use the simplest model 
holding this property

$$
H=T_{0} \sin ^{2}\left(\frac{\pi P}{P_{0}}\right)+U_{0} \sin ^{2}\left(\frac{\pi X}{a}\right),
$$

where only the lowest harmonics are considered. Here the energy of the kink is taken from its minimum classical value, i.e., the energy of a "unmoving" kink with $P=0$ or $P=P_{0}$, situated at the minimum of a pinning potential $X=0$.

For different ferromagnetic chains, any ratio of parameters $T_{0}$ and $U_{0}$ is in principle possible. For example, the value of $T_{0}$ is proportional to the difference $\left(K_{2}-K_{1}\right)$ and it vanishes in the purely uniaxial case for any value of $K_{2}=K_{1} \neq 0$, while the amplitude of the pinning potential $U_{0}$ is almost zero for $K_{1}<0.15 \mathrm{~J}$. It is natural to start with the Bohr-Sommerfeld quantization for the domain wall motion, which is based on the analysis of the classical dynamics. This can be done in the same way as for the transverse-field Ising model (see Ref. 22) and we do not discuss its details here. The most important feature of a Hamiltonian of the type (20) is the presence of a lower and upper bound for the energy. Hence, two types of finite motion appear. A first type corresponds to oscillations of the domain wall with either $P \ll P_{0}$ or $\left(P-P_{0}\right) \ll P_{0}$ near the minimum of the potential, with the energy near the minimum of the Hamiltonian, $E \ll T_{0}, U_{0}$. A second type of finite motion corresponds to oscillations near the potential maximum, with the momentum near the values of $P= \pm P_{0} / 2$, the values of energy are $E \leq T_{0}+U_{0}$.

For intermediate values of the energy, the motion is infinite. For a small pinning potential, this motion is standard, with infinite grow of the kink coordinate. The case $U_{0}>T_{0}$ is less standard; it corresponds to an infinite-growing momentum with finite oscillations of the coordinate near certain positions, which do not coincide with extrema of the pinning potential. The late case is nothing but Bloch oscillations in the pinning potential $U(X)$. An exception is the chosen point $T_{0}=U_{0}$, for which the classical motion is finite for all values of the energy.

Both types of infinite motion describe the classical over-barrier dynamics of the domain walls. Using a quantum-mechanical language, such states of the nearly-free particle can be well described by perturbation theory over $U_{0}$ or $T_{0}$, for the cases $U_{0}<T_{0}$ or $U_{0}>T_{0}$, respectively. This analysis will be done in the next subsection 【IA.

Within the Bohr-Sommerfeld quantization condition, all the states corresponding to finite motion (oscillations) of the domain wall near any extrema of the Hamiltonian produce a discrete spectrum of energy levels $E_{n}$ with a level separation of the order of $\sqrt{U_{0} T_{0}} / S$. Both 
$U_{0}$ and $T_{0}$ are proportional to $S^{2}$, therefore for the semiclassical situation of high spins $S \gg 1$, the separation of values of $E_{n} \propto S$ and it can be smaller than $U_{0}$ or $T_{0}$. These states, with energy $E_{n}$, are well localized. For them, the probability of tunneling is small, and for its estimate the semiclassical approximation is adequate. Such analysis will be done in subsection $\Pi$ IIB.

\section{A. Perturbative analysis}

For extremely large or small values of $U_{0} / T_{0}$ (namely, for $U_{0} / T_{0}<1 / S^{2}$ or $T_{0} / U_{0}<1 / S^{2}$ ) the value of $E_{n+1}-E_{n} \sim \hbar \omega_{n}$ can be of the order of $\min \left[T_{0}, U_{0}\right]$. In these cases, all the states are delocalized, and our perturbation theory gives the full description of the domain wall spectrum. Out of these strong inequalities, a perturbative analysis can only be applied to domain wall states with intermediate values of the energy, which correspond to the classical infinite motion discussed above.

Let us now start with the case $U_{0} \ll T_{0}$, using perturbation theory with respect to $U(X)$. In this case, in zeroth approximation, $P=$ const. To proceed further, let us assume the chain to have a large but finite size $L=N a, N \gg 1$. Then periodic boundary conditions give the usual quasi-continuous spectrum of the momentum $P=P_{n}=(2 \pi \hbar / a)(n / N)$, where $n$ is an integer, $N / 2<n<N / 2$, or $-P_{B} / 2<P<P_{B} / 2$, where $P_{B}=(2 \pi \hbar / a)$ is the size of the usual (crystalline) Brillouin zone. The vector of states $|P\rangle$ corresponds to a fixed value of momentum and, hence, the fixed value of chirality and the uncertainty value of the kink coordinate. The quantum spectrum of the problem repeats the dependence of the Hamiltonian (20) on $P$.

We now consider the term $U(X)$ as a perturbation. Its role will generally be the same as for the lattice potential $U(X)$ in the standard weak-binding approximation in solid state physics. At zeroth order approximation in the coordinate space, the eigen-functions are of the form $\psi^{(0)}=\exp (i P X)$, with the energy $E^{(0)}(P)=T(P)$. The influence of the potential $U(X)$ with the period $a$ leads to the formation of Bloch states which are a superposition of the states $\psi^{(n)}=\exp \left(i P X+i n P_{B}\right)$, and the momentum transforms to quasi-momentum. In the weak-binding approximation, the spectrum can be obtained by a superposition of unperturbed dispersion curves $E^{(0)}=T(P)$, with argument shifting by $n P_{B}$, where $n$ is an integer number. This spectrum is periodic with the period equal to the size of the first 
Brillouin zone $P_{B}$. The influence of the perturbation is maximal if the values of the functions $\left(P+n P_{B}\right)$ and $\left(P+n^{\prime} P_{B}\right)$, with the different $n \neq n^{\prime}$, coincide for some value of $P$.

In contrast with Bloch electrons with a parabolic dispersion law $E^{(0, e l)}=P^{2} / 2 M$, for kinks in ferromagnets, the unperturbed dispersion law is already described by a periodic function. Hence, for the resulting dispersion law $E(P)$, the periodic dependence (with the period matched with both characteristic values $P_{0}=2 \pi S \hbar / a$ and $P_{B}=2 \pi \hbar / a$ ) should appear. It is also important that kinks for the states $|P\rangle$ and $\left|P+P_{0}\right\rangle$ have the same energy and velocity, but differs by the sign of the chirality $\chi= \pm 1$. In pure classical language, these states are described by different magnetization distributions, their images corresponding to diametrically opposite paths on the sphere in Fig. 2 .

A simple analysis shows the fundamental difference between the character of the spectrum for integer and half-integer values of the atomic spin $S$. For minimal integer $S=1$, the periods $P_{0}$ and $P_{B}$ coincide: see Fig. 5. The accounting of the potential $U(X)$ of the form (20) leads to the overlapping of functions $E^{(0)}(P)$ and $E^{(0)}\left(P+P_{0}\right)$. Taking, for definiteness, a $P$ situated in the first Brillouin zone, $-P_{0} / 2<P \leq P_{0} / 2$, we can say that these unperturbed states have a different chirality $\chi=+1$ and $\chi=-1$. The action of the potential leads to their hybridization and formation of the states $|P \pm\rangle=\left(|P\rangle \pm\left|P+P_{0}\right\rangle\right) / \sqrt{2}$, having energies

$$
E^{( \pm)}(P)=E^{(0)}(P) \pm\left\langle P|U(X)| P+P_{0}\right\rangle
$$

where

$$
\begin{aligned}
& E^{(0)}(P)=T(P)+\langle U(X)\rangle, \\
& \left.\left\langle P|U(X)| P+P_{0}\right\rangle\right)=U_{0} / 4
\end{aligned}
$$

and

$$
\langle U(X)\rangle \equiv\langle P|U(X)| P\rangle)=U_{0} / 2
$$

are the off-diagonal matrix element and the mean value of the potential $U(X)$ for (20), respectively.

The same expression can be found for any integer spins $S=k>1$, i.e. the Fourier component of the potential with $P=k P_{B}=P_{0}$ leads to a full hybridization of the chirality for any $P$. As a result, states of type $|+\rangle \pm|-\rangle$ appear, where $| \pm\rangle$ correspond to chirality values $\chi= \pm 1$. Such states are the quantum superposition of the kinks describing diametrically opposite trajectories on the sphere and energy $E^{( \pm)}(P)=E^{(0)}(P) \pm U_{0} / 4$, where $U_{0}$ is the 


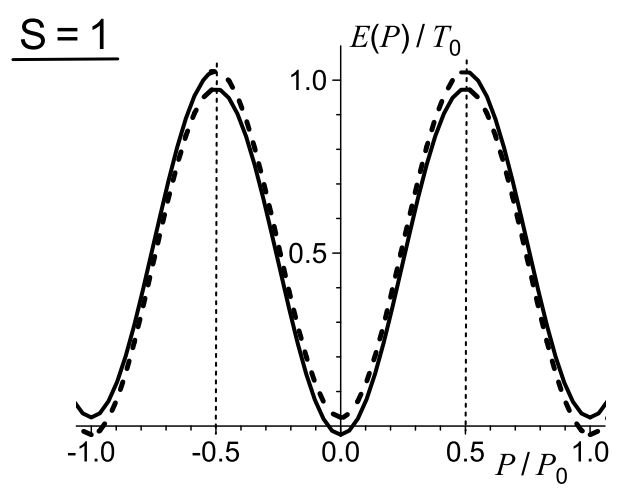

FIG. 5: Dispersion relation of a kink in a ferromagnet with spin $S=1$ subject to a weak pinning potential. The solid line and the dashed line correspond to the states $(|+\rangle-|-\rangle)$ and $(|+\rangle+|-\rangle)$ , antisymmetric and symmetric over chirality, respectively. Here, and in the Figs. 66 8, the vertical dotted lines show the boundaries of the usual Brillouin zone, $-P_{B} / 2<P \leq P_{B} / 2$.

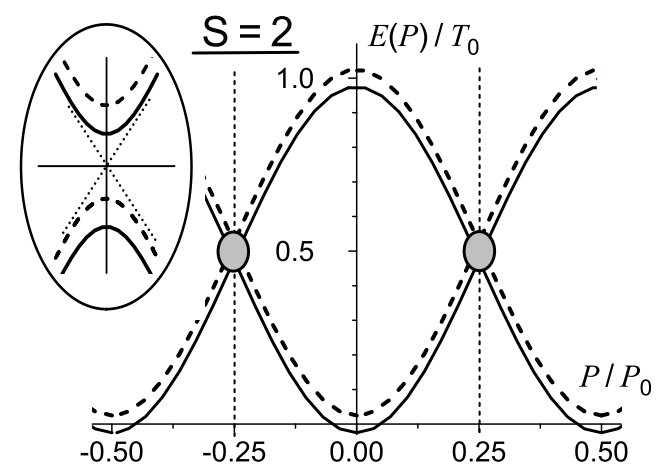

FIG. 6: Same as for Fig. 5 for spin value $S=2$. The region near the crossing points, schematically shown by shadowed ellipses on the main figure, are magnified in the insert upward left.

corresponding matrix element of the potential $U(X)$. For integer spins $S>1$, the value $P_{0}=S \cdot P_{B}$ is a common period. Neglecting the chirality tunneling, one can find the $S$ usual energy bands (doubly degenerated over the chirality values) with the size of $P_{B}$. The chirality tunneling splits any of them into two subbands, corresponding to states $(|+\rangle \pm|-\rangle)$ with $E^{( \pm)}(P)$, and the total number of bands equals to $2 S$, as shown in Fig. 6 ,

For half-integer spins $S=k+1 / 2$, where $k$ is an integer number, the situation is completely different. It is easy to show that none of the Fourier components of the potential with $n P_{B}=2 n P_{0} /(2 k+1)$ leads to such an overlapping of the non-perturbed spectrum at 


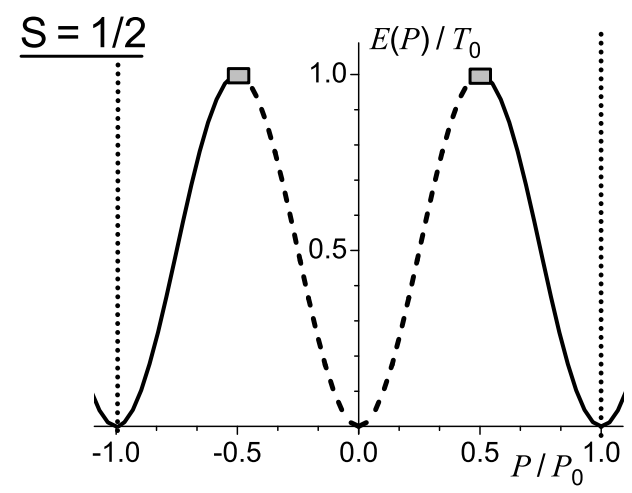

FIG. 7: Dispersion relation of a kink in a ferromagnet with spin $S=1 / 2$, subject to a weak pinning potential. The solid line and dashed line correspond to the states with chirality $\chi=-1$ and $\chi=1$, respectively. Rectangles denote the points where the value of the chirality is not determined.

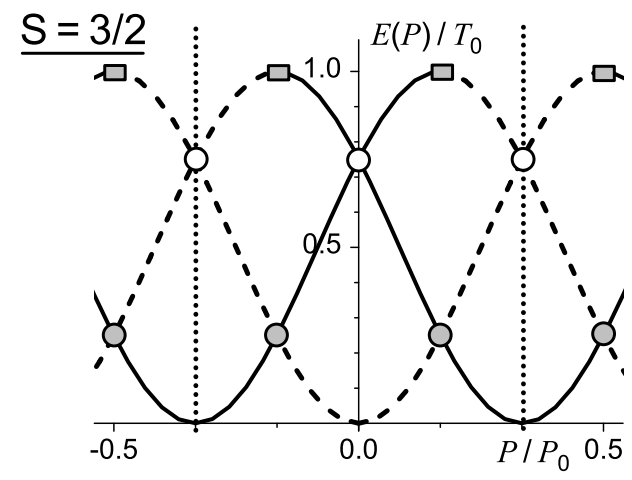

FIG. 8: Same as in Fig. 7 for the spin value $S=3 / 2$. The gray and the light circles schematically show the areas in the vicinity of the crossing of unperturbed spectra with and without chirality hybridization, respectively.

any $P$, which take place for integer $S$. For $S=1 / 2$ any crossing is absent, as shown in Fig. 7 [a crossings of non-perturbed spectra for spin $S=1 / 2$ reported in Ref. 27 is an artifact of the parabolic approximation for $E^{(0)}(P)$ ]. For higher half-integer spins $S>1 / 2$ the only crossings at some fixed values occur when $P=P_{n}$. Such crossings can appear for branches $E^{(0)}\left(P+n P_{B}\right)$, corresponding to the kinks with the same or different chirality, as shown in Fig. 8, For this last case, the effects of chirality hybridization can be present very near the crossing points, $\left|T(P)-T\left(P_{n}\right)\right| \ll U_{0}$, as shown in Fig. 8 .

Let us consider the opposite limiting case $U_{0} \gg T_{0}$, when the kinetic energy $T(P)$ plays 
the role of a small perturbation for the unperturbed Hamiltonian $H_{0}=U(X)$. To zeroth order approximation with respect to $T_{0}$, the solution is now $X=$ const. To construct this perturbation theory, the momentum representation should be used. In this case, taking into account the identity of the states with $P$ and $P+2 P_{0}$, we should apply the condition $\exp \left[i\left(P-P_{0}\right) X\right]=\exp \left[i\left(P+P_{0}\right) X\right]$, that leads to the discreteness of the values of the kink coordinate, $X=X_{k}=a k / 2 S$. The difference between this boundary condition and the condition which was used for the case $U_{0} \ll T_{0}$ above, leads to essentially different results.

For the case of strong pinning potential $U_{0} \gg T_{0}$, unperturbed states are described by the wave function in the momentum representation $\psi^{(0)}=\exp \left(i X_{k} P\right)$, with definite coordinate $X=X_{k}$ and indefinite value of the momentum, which also means an indefinite value of the chirality. Let us now consider $T(P)$ as a small perturbation. It is easy to see that $n$th Fourier component of $T(P)$, with $\exp \left(2 i \pi n P / P_{0}\right)$, leads to a nonzero matrix element of the quantum transition when changing the kink coordinate, $X_{k} \rightarrow X_{k}+\delta X_{n}, \delta X_{n}=n a / S$ (in the simplest case with one harmonic only, Eq. (20), the transition with $n>1$ requires accounting the $n$th order in perturbation theory). If $U\left(X_{k}\right)=U\left(X_{k}+\delta X_{n}\right)$, i.e. when the value of $\delta X_{n}$ is multiple to the chain period $a$, the "resonant" transition should be observed.

Summarizing, kinks in ferromagnetic chains with either integer or half-integer spins behave differently. The tunneling from a cell to the neighboring one is possible for an integer spin, while for the half-integer spin only the transitions with changing $X$ by $2 a$ are permitted, i.e. "jumps" across one cell. This feature was mentioned in Ref. 27 using a different reasoning.

\section{B. Semiclassical dynamics}

For large values of the spin, $S \gg 1$, a semiclassical approach provides a much better approximation than the perturbative approach used above. For electronic states in a large lattice potential, the semiclassical tunneling can be formally described by the tight-binding approximation, which leads to the formation of a band spectrum of the kink with narrow allowed bands.

Considering possible tunneling effects for a kink, one immediately encounters the question of under-barrier transition in both coordinate space and momentum space. For domain walls with the minimal energy, such transitions include the tunneling between states cor- 
responding to a two-dimensional set of points in phase space of a system $(P, X)$, such as $X \simeq 0, \pm a, \ldots$ and $P \simeq 0, \pm P_{0}, \ldots$ Within the semiclassical approximation, these transitions can be investigated in the framework of the instanton approach; see Refs. 36,38. This approach is a version of the Feynman path integral method suited to the description of the underbarrier transitions. It involves using the Euclidean space-time, that is transforming to the imaginary time, $t \rightarrow i \tau$ (so called Wick rotation). Within this approach, the amplitude of the underbarrier transition from a given quantum state $|i\rangle$ to another one $|f\rangle$ is determined by the path integral $\int D X \cdot \exp \left[-A_{E}[X] / \hbar\right]$, where $D X$ denotes integration over all possible paths that satisfy the specified boundary conditions. Here the Euclidean action $A_{E}[X]$ is described in the form $A_{E}=\int L_{E} d \tau$, and $L_{E}=P(d X / d \tau)-H(P, X)$ is obtained by the application of a Wick rotation to the usual mechanical Lagrangian. The instanton solution determines the trajectory for which the tunneling amplitude is maximal, i.e.; the instanton trajectory minimizing $A_{E}$ with respect to $X(\tau)$ and $P(\tau)$, with the conditions $|i\rangle$ at $\tau \rightarrow-\infty$ and $|f\rangle$ at $\tau \rightarrow+\infty$. The minimum of the Euclidean action is realized on the separatrix solution of the corresponding Euler-Lagrange problem for the Euclidean action functional $A_{E}$ or, equivalently, on the solution of the Hamilton equation with the substitution $t \rightarrow i \tau$. The tunneling splitting of the levels $\Delta$ is determined by the formula $\Delta=\eta \hbar \omega_{0} \sqrt{A_{E} / \hbar} \exp \left(-A_{E}^{0} / \hbar\right)$, where $\omega_{0}$ is the characteristic frequency, $\eta \sim 1$.

Let us apply this approach to the mechanical problem of the kink dynamics described by the Hamiltonian (20). It is easy to see that the Wick rotation $t \rightarrow i \tau$, simultaneously with the simple substitution $X \rightarrow i \Xi$, reduces the instanton problem to the Hamilton problem for real canonical variables $\Xi$ and $P$ and with the real Hamilton function $H_{E}$,

$$
H_{E}=T_{0} \cdot \sin ^{2}\left(\pi P / P_{0}\right)-\mathcal{U}(\Xi), \mathcal{U}(\Xi)=U_{0} \cdot \sinh ^{2}(\pi \Xi / a)
$$

The Hamilton equations for (21) have an obvious integral of motion $H_{E}=$ const; boundary conditions give $H_{E}=0$. Thus, for an instanton solution, we derive $\sqrt{T_{0}} \sin \left(\pi P / P_{0}\right)=$ $\pm \sqrt{\mathcal{U}(\Xi)}$. A simple analysis shows that this problem has an instanton solution with $\Xi( \pm \infty)=0$ (i.e., $X( \pm \infty)=0$ ), while the values of momentum differ: $P$ at $\tau \rightarrow-\infty$ and $P+P_{0}$ at $\tau \rightarrow+\infty$. This instanton solution describes the tunneling of the kink chirality.

Analysis of the second type of tunneling (tunneling of the kink coordinate) can be done in a similar manner, by using a Wick rotation, substituting $P \rightarrow i \Pi$, and keeping the 


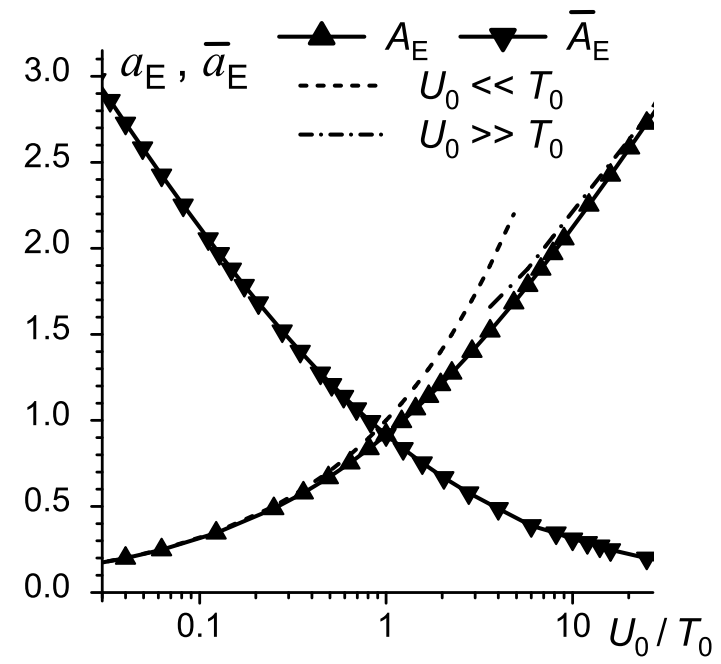

FIG. 9: Dependence on the parameters $a_{E}$ and $\bar{a}_{E}$, determining the value of the Euclidean action for the tunneling processes, as a function of $U_{0} / T_{0}$ (in logarithmic scale). Two analytic asymptotic dependencies are also indicated in the figure with a dashed and a dot-dashed curves.

coordinate $X$ as a real variable. Then, again, the real Hamilton function $\bar{H}_{E}$ for two real variables $X, \Pi$ appears,

$$
\bar{H}_{E}=U_{0} \cdot \sin ^{2}(\pi X / a)-T_{0} \cdot \sinh ^{2}\left(\pi \Pi / P_{0}\right) .
$$

It is easy to show that this problem has an instanton solution with $\Pi( \pm \infty)=0, X(\tau \rightarrow$ $-\infty)-X(\tau \rightarrow \infty)=a$. This instanton solution describes the tunneling of the kink from one lattice site to a neighboring one.

For the instanton solutions of both types $\bar{H}_{E}, H_{E}=$ const $=0$ the Euclidean Lagrangian reduces to $L_{E}=P(d X / d \tau)$. Therefore for both cases, (21) and (22), the value of the Euclidean action can be represented as simple integrals. For example, for the tunneling of the chirality $A_{E}^{0}=\int_{0}^{P_{0}} \Xi(P) d P$, where

$$
\Xi(P)=(a / \pi) \operatorname{arcsinh}\left[\sqrt{\left(T_{0} / U_{0}\right)} \sin \left(\pi P / P_{0}\right)\right],
$$

resulting in

$$
A_{E}^{0}=\left(\frac{2 \hbar S}{\pi}\right) a_{E}\left(\frac{U_{0}}{T_{0}}\right)
$$

where $a_{E}\left(U_{0} / T_{0}\right)$ is a universal function (see Fig. 9) that only depends on the ratio $U_{0} / T_{0}$. We can easily find the asymptotic behavior of $a_{E}$ : the functions $a(z)=\sqrt{z}$ and $a(z)=\ln z$, respectively, where $z=U_{0} / T_{0}$. These are shown in Fig. 9. 
For the analysis of the tunneling of the coordinate $X$ it is not even necessary to calculate the corresponding integral $\bar{A}_{E}^{0}=\int_{0}^{a} \Pi(X) d X$. The Euclidean action $\bar{A}_{E}$, describing the tunneling of the kink coordinate, is obtained from the previous formula for the tunneling of the chirality $A_{E}$ in (23) by the replacement $U_{0} / T_{0} \rightarrow T_{0} / U_{0}$.

The quantity $A_{E}$, as well as the tunneling splitting $\Delta_{0}$, is a standard measure of the intensity of the quantum tunneling processes; but there is a physical difference among the tunnel processes considered here. This is because all of the values of the kink coordinate of the form $X=$ an (the kink is found at points of potential energy minima) correspond to different states of the kink, while all the values of the momentum differing by $2 P_{0}$ correspond to the same state of the kink. Therefore, the processes of tunneling either coordinate or momentum lead to different physical consequences. Tunneling of the coordinate is responsible for the formation of an energy band in which the number of states with different values of the quasi-momentum coincides with the number of spins in the chain. The width of the corresponding energy band is given in terms of the tunneling splitting $\Delta_{0}, \Delta E_{0}=2 \Delta_{0}$. Tunneling of the momentum leads to lifting off the twofold degeneracy of the states of the kink and causes a splitting of the level into two, with $\Delta E=2 \bar{\Delta}_{0}$

\section{CONCLUDING REMARKS AND RESULT DISCUSSION}

In conclusion, using both semiclassical instanton and perturbative approaches, we study quantum tunneling effects in $P$-space and $X$-space for domain walls in ferromagnetic chains. We also investigated the quantum dynamics of domain walls (kink-type solitons) in spin chains. Explicit results have been obtained for the biaxial model with isotropic interaction $J$, and rhombic anisotropy with two constants $K_{1}$ and $K_{2}$. The combinations of the two spin interaction constants, $K_{1} / J$ and $\left(K_{2}-K_{1}\right) / K_{1}$, define two parameters, $U_{0}$ and $T_{0}$, for the effective Hamiltonian describing the quantum dynamics of a domain wall.

In both limits (small and large $U_{0} / T_{0}$ ) only one type of transition becomes important, but for the case $U_{0} \sim T_{0}$ the probability for both transitions are comparable. In this case $U_{0} \sim T_{0}$, the function $a\left(U_{0} / T_{0}\right)$ is of order of unity, and for $S \sim 1$ both transition amplitudes are not small; i.e., the kinks in ferromagnetic chains with spin $S \sim 1$ are essentially quantum objects. They are characterized by a quantum dispersion relation (spectrum) of the kink $E=E(P)$, with the presence of some discrete variable, chirality $\chi= \pm 1$. The quantum 
properties of domain walls in a chain with either integer or half-integer spin are essentially different. For a chain with integer spin, there are $S$ main energy bands, each one of them split in two subbands, with a total hybridization of the chirality. In contrast, for the case of half-integer spin chain we arrive at a pattern of $2 S$ nonoverlapping energy bands, with chirality hybridization only at some particular points.

Having in mind the case of mesoscopic chain-like artificial ferromagnetic structures, we discuss the behavior of domain walls for large spins $S \gg 1$. At a first glance, for such systems, the quantum effects should be suppressed by the large spin values. However, as we have shown, tunneling effects can occurs, even for values like $S \sim 10^{2}-10^{3}$, for essentially different values of the parameters $U_{0}$ and $T_{0}$. Here the value of the tunneling exponent can be acceptable $\left(A_{E} / \hbar\right) \leq 15-20$ if $a_{E} \ll 1$. We stress the agreement between the probability

of tunneling for one mesoscopic magnetic particle, see Refs. 37,38 and the probability of tunneling processes for the kinks found here.

For domain wall tunneling, at least one of two quantum tunneling transitions (either tunneling of coordinates or tunneling of chirality) are possible. It is useful to introduce the following empiric rule. The probability of chirality tunneling, including flipping a large number of spins $N_{\text {kink }} \gg 1$ has the same order of magnitude as for coherent spin tunneling of a single particle.

\section{Acknowledgments}

We gratefully acknowledge partial support from the National Security Agency (NSA), Laboratory Physical Science (LPS), Army Research Office (ARO), National Science Foundation (NSF) grant No. EIA-0130383, JSPS-RFBR 06-02-91200, and Core-to-Core (CTC) program supported by Japan Society for Promotion of Science (JSPS). SS acknowledges support from the Ministry of Science, Culture and Sport of Japan via the Grant-in Aid for Young Scientists No 18740224, the EPSRC via No. EP/D072581/1, EP/F005482/1, and ESF network-programme "Arrays of Quantum Dots and Josephson Junctions".

* Electronic address: bivanov@i.com.ua

1 A. Hubert and R. Schäfer, Magnetic Domains (Springer-Verlag, Berlin, 1999). 
2 A.P. Malozemoff and J.C. Slonczewski, Magnetic Domain Walls in Bubble Materials (Academic Press, New York, 1979).

3 V. G. Baryakhtar, M. V. Chetkin, B. A. Ivanov and S. N. Gadetskii, Dynamics of topological magnetic solitons. Experiment and theory, Springer Tract in Modern Physics 139, SpringerVerlag, Berlin, (1994).

4 H.-J. Mikeska and M. Steiner, Adv. Phys. 40, 191 (1991).

5 V. G. Bar'yakhtar and B. A. Ivanov, Solitons and Thermodynamics of Low-Dimensional Magnets in Soviet Scientific Reviews, Section A. Physics, I. M. Khalatnikov (ed.), 16 (1992)

6 A. M. Kosevich, B. A. Ivanov, and A. S. Kovalev, Phys. Rep. 194, 117 (1990).

7 R. Skomski, J. Phys.: Condens. Matter 15, R841 (2003).

8 W. Wernsdorfer, Adv. Chem. Phys. 118, 99 (2001).

9 R. P. Cowburn and M. E. Welland, Science 287, 1466 (2000).

10 S. Savel'ev, A. L. Rakhmanov, F. Nori, Phys. Rev. B 74, 024404 (2006); S. Savel'ev, A. Rakhmanov, F. Nori, New J. Phys. 7, 82 (2005).

11 R. Antos, Y. Otani, J. Shibata, J. Phys. Soc. Jpn., in press.

12 J.I. Martin et al., J. Magn. Magn. Mater. 256, 449 (2003); S.O. Demokritov et al., Phys. Rep. 348, 441 (2001); S.D. Bader, Rev. Mod. Phys. 78, 1 (2006); G. Srajer et al., J. Magn. Magn. Mater. 307, 1 (2006).

13 M. Hara, T. Kimura, Y. Otani, Appl. Phys. Lett. 90, 242504 (2007).

14 M. Hara, J. Shibata, T. Kimura, Y. Otani, Appl. Phys. Lett. 89, 192504 (2006); A. Yamaguchi et al., Phys. Rev. Lett. 92, 077205 (2004).

15 L.N. Leuenberger, D. Loss, Nature 410, 789 (2001); W. Wernsdorfer, N. Aliaga-Alcalde, D. N. Hendrickson, G. Christou, Nature 416, 406 (2002).

16 B.A. Ivanov, Fiz. Nizk. Temp. 31, 841 (2005).

17 B.A. Ivanov and A.K. Kolezhuk, JETF Lett. 60, 792 (1994); Phys. Rev. Lett.74, 1859 (1995).

18 B. A. Ivanov, A. K. Kolezhuk and V.E. Kireev, Phys. Rev. B58, 11514 (1998).

19 S. Savel'ev, A.L. Rakhmanov, F. Nori, Phys. Rev. Lett. 98, 077002 (2007); Phys. Rev. Lett. 98, 269901(E) (2007); A. O. Sboychakov, S. Savel'ev, A. L. Rakhmanov, F. Nori, Europhys. Lett. 80, 17009 (2007); P. Hänggi, F. Marchesoni, and P. Riseborough, Europhys. Lett. 13, 217 (1990); P. Hänggi, P. Talkner, and M. Borkovec, Rev. Mod. Phys. 62, 251 (1990).

20 S. Savel'ev, A. L. Rakhmanov, X. Hu, A. Kasumov, F. Nori, Phys. Rev. B 75, 165417 (2007); 
S. Savel'ev, X. D. Hu, and F. Nori, New J. Phys. 8, 105 (2006).

21 X. Hu, F. Nori, Phys. Rev. Lett. 79, 4605 (1997); X. Hu, F. Nori, Phys. Rev. Lett. 76, 2294 (1996); X. Hu, F. Nori, Phys. Rev. B 53, 2419 (1996); X. Hu, F. Nori, Physica B 263, 16 (1999).

22 B. A. Ivanov and H.-J. Mikeska, Phys. Rev. B70, 174409 (2004).

23 E. Fradkin, Field theories of condensed matter systems, in Frontiers in Physics, $\mathbf{8 2}$ (AddisonWesley, 1991).

24 A. I. Akhiezer, V. G. Bar'yakhtar, and S. V. Peletminskii, Spin Waves (North-Holland, Amsterdam, 1968).

25 D. Loss, D. P. DiVincenzo, and G. Grinstein, Phys. Rev. Lett. 69, 3232 (1992).

26 J. von Delft and C. L. Henley, Phys. Rev. Lett.69, 3236 (1992).

27 H.-B. Braun and D. Loss, Phys. Rev. B 53, 3237 (1996).

28 S. Takagi and G. Tatara, Phys. Rev. B 54, 9920 (1996).

29 J. Shibata and S. Takagi, Phys. Rev. B 62, 5719 (2000).

30 B. A. Ivanov, A. Yu. Merkulov, V. A. Stephanovich, and C. E. Zaspel, Phys. Rev. B 74, 224422 (2006).

31 A. N. Goncharuk, A. A. Stepanov, and D. A. Yablonskii, Sov. Phys. Solid State 31, 2099 (1989);

M. V. Gvozdikova, A. S. Kovalev, and Yu. S. Kivshar, Low Temp. Phys. 24, 479 (1998).

32 E. G. Galkina and B. A. Ivanov, Fiz. Nizk. Temp. 33, 601 (2007).

33 E. G. Galkina and B. A. Ivanov, JETP Lett. 71, 259 (2000).

34 G.E. Volovik and V.P. Mineev, JETP 72, 2256 (1977).

35 D. Mermin, Rev. Mod. Phys. 51, 591 (1979).

36 A.I. Vainshtein, V.I. Zakharov, V.A. Novikov, and M.A. Shifman, Usp. Fiz. Nauk 136, 553 (1982)[Sov. Phys. Usp. 24, 195 (1982)].

37 E. M. Chudnovsky and J. Tejada, Macroscopic Quantum Tunneling of the Magnetic Moment (Cambridge University Press, Cambridge, 1998).

38 Quantum Tunneling of Magnetization, L. Gunter and B. Barbara (editors), NATO ASI Series E, vol. 301, Kluwert, Dordrecht, (1995). 